\title{
Angeborene Störungen der Lungen und Atemwege - Übersicht der Krankheitsfälle der Kinderklinik Erlangen aus den Jahren 2000 bis 2011*
}

\author{
Congenital Disorders of the Lungs and Airways - \\ Survey of Cases at the Paediatric Hospital Erlangen in the Period 2000 to 2011
}

Autoren

Institut
S. Meyer, T. Zimmermann

Klinik für Kinder und Jugendliche der Friedrich-Alexander-Universität Erlangen-Nürnberg, Erlangen eingereicht $\quad 28.12 .2012$ akzeptiert nach Revision 16.4.2013

\section{Bibliografie}

Dol http://dx.doi.org/ 10.1055/s-0033-1344124 Online-Publikation: 5.6.2013 Pneumologie 2013; 67: 388-397 (c) Georg Thieme Verlag KG Stuttgart · New York ISSN 0934-8387

Korrespondenzadresse Sarah Meyer Lortzingstraße 28 90571 Schwaig bei Nürnberg sarahmeyer_sm@web.de

\section{Zusammenfassung \\ V}

Hintergrund: Kongenitale Lungenfehlbildungen sind selten, können aber die vitalen Funktionen der Betroffenen stark beeinträchtigen. Mit Hilfe der in den letzten Jahrzehnten zunehmenden diagnostischen und therapeutischen Möglichkeiten können diese Erkrankungen jedoch immer früher festgestellt und erfolgreich behandelt werden. Der folgende Artikel gibt einen Überblick über die in der Kinderklinik Erlangen aufgetretenen Fälle an Lungenfehlbildungen in den Jahren 2000 bis 2011, über assoziierte Missbildungen sowie über diagnostische und therapeutische Maßnahmen. Methode: Die Analyse erfolgte retrospektiv mit Hilfe der Krankenakten der Patienten. Grundlage waren Daten von Kindern, die in dem Zeitraum vom 1.1.2000 bis 15.4.2011 an der Kinderklinik Erlangen stationär bzw. teilstationär aufgrund einer Lungen- bzw. Atemwegsfehlbildung therapiert wurden. Ausgehend von den jeweiligen ICD-Codierungen wurden zunächst Namenslisten der Patienten erstellt und anschließend deren Daten ausgewertet und mit Literaturangaben verglichen. Ergebnisse: In dem Zeitraum vom 1.1.2000 bis 15.4.2011 wurden 121 Kinder aufgrund einer angeborenen Lungen- bzw. Atemwegsfehlbildung in Erlangen diagnostiziert und therapiert. $68,6 \%(n=83)$ der Kinder waren männlich und 31,45\% ( $n=38)$ weiblich. Bei fast allen Patienten waren die Anomalien mit weiteren Fehlbildungen, wie angeborenen Herzfehlern, assoziiert. 28 der 121 beschriebenen Kinder litten an mehr als einer kongenitalen Lungenfehlbildung. In Bezug auf die diagnostischen und therapeutischen Maßnahmen entsprach das Vorgehen der Erlanger Pädiater weitestgehend den in der Literatur empfohlenen Interventionen. Praktische Schlussfolgerungen: Die für diese Arbeit durchgeführte Analyse der Krankenakten zeigt die Wichtigkeit einer frühzeitigen Diagnosestellung und Therapie bei angeborenen Lungen- bzw. Atem-

\section{Abstract \\ V}

Background: Congenital malformations of the lungs are rare, but might limit the quality of life of the patient. With the increased possibilities of diagnostic and therapeutic procedures during the last decades, these diseases can be recognised and successfully treated earlier than before. The aim of this article is to give an overview about the congenital malformations of the lungs in children who have been treated at the Kinderklinik Erlangen during the years 2000 and 2011. Clinical symptoms of the disease and associated malformations, diagnostic and therapeutic procedures were discussed. Methods: The analysis was done retrospectively (January $1^{\text {st }} 2000$-April $15^{\text {th }} 2011$ ) on the basis of the patients records. Patients who had been treated only at the outpatient clinic were not considered. All patients' data were listed according to the ICD code. Results: 121 children were treated because of a congenital malformation of the lungs in the years between 2000 and 2011 in Erlangen. 68.6\% ( $n=83)$ were males and $31.45 \%(n=38)$ were females. Nearly all of the malformations of the lungs were associated with other abnormalities. Most of the children also suffered from at least one cardiac defect. 28 of the 121 children had more than one malformation of the lungs. The diagnostic and therapeutic interventions correlated with the published recommendations. Conclusions: The analysis of the patients' data used in this study indicate the importance of the early diagnosis and treatment. The cooperation of different medical disciplines is mandatory for a good outcome of those children.

\footnotetext{
* Das Manuskript stellt Ergebnisse eines Promotionsvorhabens aus der Kinderklinik Erlangen der FriedrichAlexander-Universität Erlangen-Nürnberg (Direktor: Prof. Dr. med. Dr. h.c. Wolfgang Rascher) vor.
} 
wegsfehlbildungen. Durch eine interdisziplinäre klinische Zusammenarbeit konnte vielen der Patienten geholfen werden, sodass diese Kinder oft gute Chancen auf ein späteres normales Leben besitzen.

\section{Fragestellung}

In der Literatur finden sich zahlreiche Beschreibungen kongenitaler Lungen- und Atemwegsfehlbildungen mit Angaben zur Klinik, Diagnostik, Therapie und Häufigkeit. Ziel dieser retrospektiven Analyse war es, die an der Kinderklinik Erlangen aufgetretenen Fälle an angeborenen Anomalien in dem Zeitraum vom 1.1.2000 bis 15.4.2011 zu beschreiben und mit publizierten Daten zu vergleichen. Hierzu wurden neben den klinischen Symptomen und diagnostischen bzw. therapeutischen Maßnahmen auch assoziierte Missbildungen untersucht.

\section{Material und Methode}

\section{$\nabla$}

Grundlage dieser Arbeit waren Daten aus den Krankenakten von Kindern, die in dem Zeitraum vom 1.1.2000 bis 15.4.2011 an der Kinderklinik Erlangen stationär bzw. teilstationär aufgrund einer Lungenfehlbildung behandelt wurden. Nicht berücksichtigt wurden dabei ausschließlich ambulant behandelte Patienten, da diese erst seit wenigen Jahren digital erfasst werden und hierzu somit keine vollständige und statistisch relevante Aussage getroffen werden kann. Die Retrospektivanalyse erfolgte anhand des ICD-Diagnoseschlüssels, mit dessen Hilfe zunächst Namenslisten der in der Kinderklinik therapierten Patienten erstellt und anschließend die Krankenakten gesichtet wurden.

\section{Ergebnisse}

\section{$\nabla$}

In dem Zeitraum vom 1.1.2000 bis 15.4.2011 wurden 121 Kinder aufgrund einer angeborenen Lungen- bzw. Atemwegsfehlbildung in Erlangen vorgestellt und therapiert.

$68,6 \%(n=83)$ der Kinder waren männlich und 31,45\% $(n=38)$ weiblich. Die Patienten waren mit 90,9\% bei Diagnosestellung überwiegend im Neugeborenenalter.

Folgende Fehlbildungen wurden bei den Patienten diagnostiziert: Hypoplasie $(n=36)$, Tracheomalazie $(n=26)$, Trachealstenose $(n=$ 21), AV-Fistel $(n=15)$, Lungensequester $(n=14)$, Lungenzysten $(n=12)$, Trachealbronchus $(n=6)$, Bronchomalazie/Bronchusstenose $(n=6)$, Lappungsanomalien $(n=3)$, H-Fistel $(n=3)$, Primäre Ziliäre Dyskinesie $(n=3)$, Alveolokapilläre Dysplasie $(n=2)$, Bronchiektasie $(n=1)$, Williams-Campbell-Syndrom $(n=1)$, Lungenagenesie $(n=1)$, Wilson-Mikity-Syndrom $(n=1)$, Surfactant-Protein C-Defizienz $(n=1)$, hepatopulmonale Fusion $(n=1)$.

- Abb. 1 gibt einen Überblick über Anzahl und Geschlechterverteilung bei den einzelnen Krankheiten.

Bei fast allen Patienten waren die Lungen- bzw. Atemwegsanomalien mit weiteren Fehlbildungen assoziiert, wobei auffällt, dass viele der Kinder neben der Lungenanomalie zusätzlich einen Herzfehler aufwiesen.

28 der 121 beschriebenen Kinder litten an mehr als einer angeborenen Lungenfehlbildung. Beispielsweise wurde bei 6 der 14 Kinder mit einem Lungensequester zusätzlich ein Scimitar-Syndrom nachgewiesen $(42,9 \%)$.

An diagnostischen Maßnahmen wählten die behandelnden Pädiater hauptsächlich die Röntgenuntersuchung des Thorax, die Computertomografie, die Magnetresonanztomografie und/oder die Bronchoskopie.

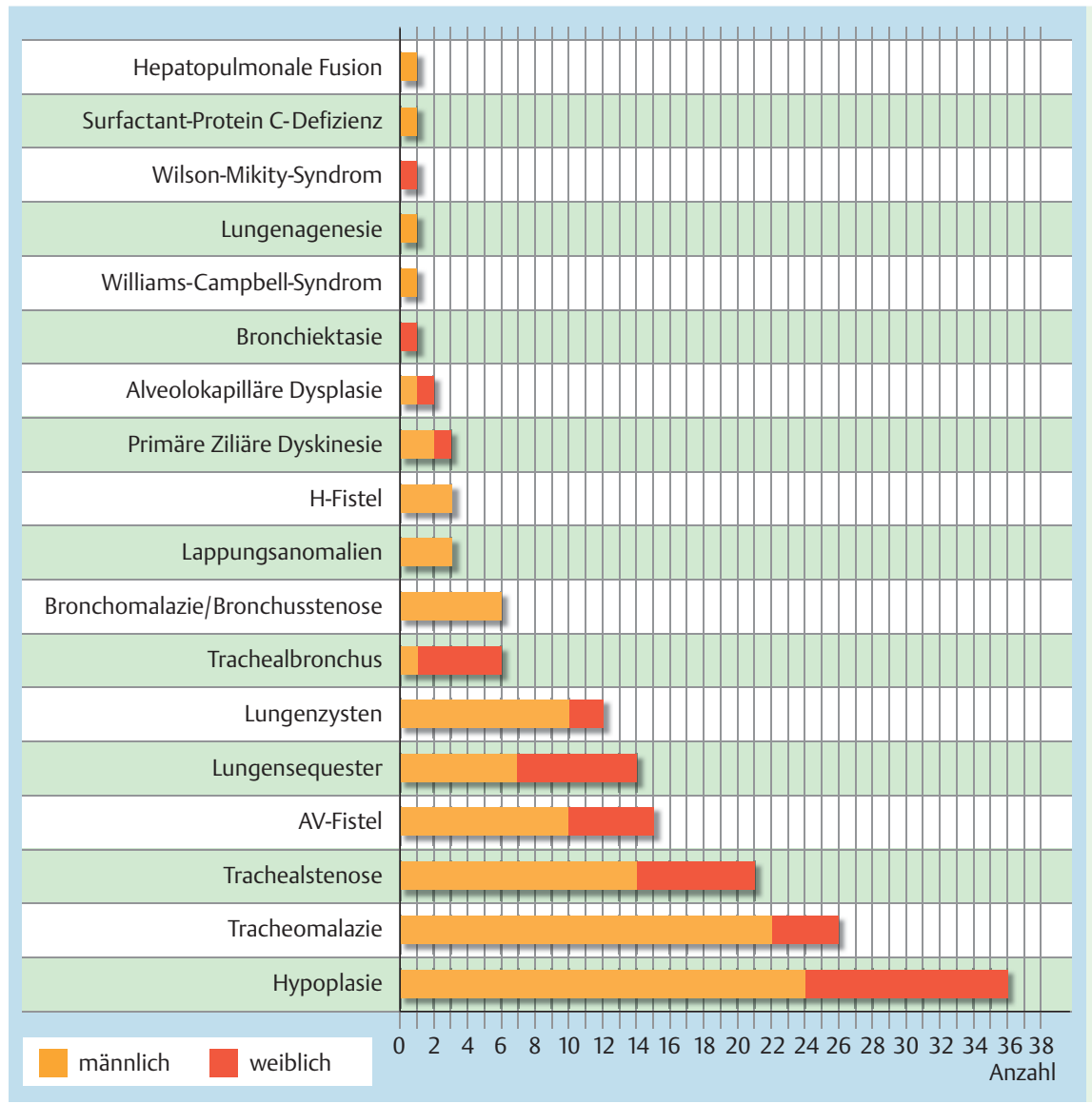

Abb. 1 Geschlechterverteilung bei den einzelnen Krankheiten. 
Im Folgenden werden einige ausgewählte Lungenfehlbildungen beschrieben und mit der Literatur verglichen.

\section{Lungensequester}

Über die Inzidenz der Lungensequestration finden sich verschiedene Angaben in der Literatur: Khalil et al. diagnostizierten in einer Fallanalyse neun Fälle innerhalb von 13 Jahren [1]. Bei den von Carter durchgeführten Lungenresektionen wurde in 1,1-1,8\% der Untersuchungen eine Lungensequestration nachgewiesen. Hierbei dominierten mit $85 \%$ die intralobären gegenüber den extralobären Sequestern [2]. Gremmel et al. diagnostizierten innerhalb eines Zeitraums von zehn Jahren bei sechs Patienten eine lobäre Sequestration [3], während Kabnick et al. von einer Inzidenz von $1,8 \%$ bis $2 \%$ aller Lungenfehlbildungen spricht [4]. In den Jahren von 1980 bis 1985 wurden laut D. Schneider insgesamt zehn Patienten aufgrund eines intralobären Sequesters in der Kinderklinik Erlangen therapiert, wobei dieser sich in $80 \%$ der Fälle in einem Lungenunterlappen befand. Dabei war bei fünf Patienten der rechte und bei drei Patienten der linke Unterlappen betroffen [5].

In dem für diese Arbeit untersuchten Patientenkollektiv wiesen 14 Kinder eine Lungensequestration auf. Bei zwei Patienten handelte es sich um einen intralobären Sequester, während ein Kind einen seltenen, subphrenischen und damit extralobären Sequester aufwies. Bei den restlichen elf Patienten war in den Krankenakten keine genauere Beschreibung des Sequesters vermerkt.

Insgesamt dominierte mit 57,2\% der rechte Unterlappen als Manifestationsort, während $14,3 \%$ der Sequestrationen im linken Unterlappen diagnostiziert werden konnten. In 7,1\% befand sich die Anomalie im rechten Oberlappen und bei ebenfalls 7,1\% handelte es sich, wie bereits erwähnt, um eine subphrenische und damit extrapulmonal lokalisierte Raumforderung. Bei den restlichen 14,3\% lag ein Sequester in der rechten Lunge vor.

In der Literatur wird von einer bevorzugten Lokalisation dorsobasal links gesprochen. Insgesamt sollen etwa $80 \%$ der Lungensequestrationen links lokalisiert sein [6-8]. Dies ließ sich durch das Erlanger Patientenkollektiv jedoch nicht bestätigen.

Die Gefäßversorgung erfolgt laut diversen Autoren zumeist aus einem aberranten Ast der Aorta und nur selten aus einer Pulmonalarterie. Dabei ist in $80 \%$ der Fälle die Aorta thoracica descendens Ursprung des Gefäßes. Bei 20\% der extralobären und 15\% der intralobären Sequester lässt sich eine Mehrfachversorgung nachweisen. Der überwiegende Anteil weist aber eine Einfachversorgung auf [9-12]. Der venöse Abfluss erfolgt normalerweise ins linke Atrium, gelegentlich auch ins rechte Atrium, zur Vena cava inferior oder Vena azygos [13 - 15]. Bei den 14 in der Kinderklinik Erlangen diagnostizierten Sequestern wiesen 57,2\% eine arterielle Einfachversorgung auf, während bei $14,3 \%$ eine Mehrfachversorgung nachgewiesen werden konnte. Dabei dominierte mit jeweils 37,5\% eine aberierrende Arterie aus der Aorta descendens bzw. Aorta abdominalis, während der Sequester bei $7,1 \%$ aus der Aorta ascendens versorgt wurde. Bei den restlichen Kindern konnte die Gefäßversorgung nicht eindeutig festgestellt werden. Dies deckt sich weitgehend mit den in der Literatur vorgefundenen Informationen bezüglich einer Dominanz der Aorta descendens als Ursprung. Im Hinblick auf die venöse Drainage konnte bei 14,3\% ein Abfluss über die Lungenvenen in den linken Vorhof und bei 7,1\% über eine Scimitarvene angiografisch sichergestellt werden. In 78,6\% der Fälle konnte der venöse Blutabfluss nicht zweifelsfrei bestimmt werden. $\bullet$ Abb. 2 zeigt eine CT-Auf-

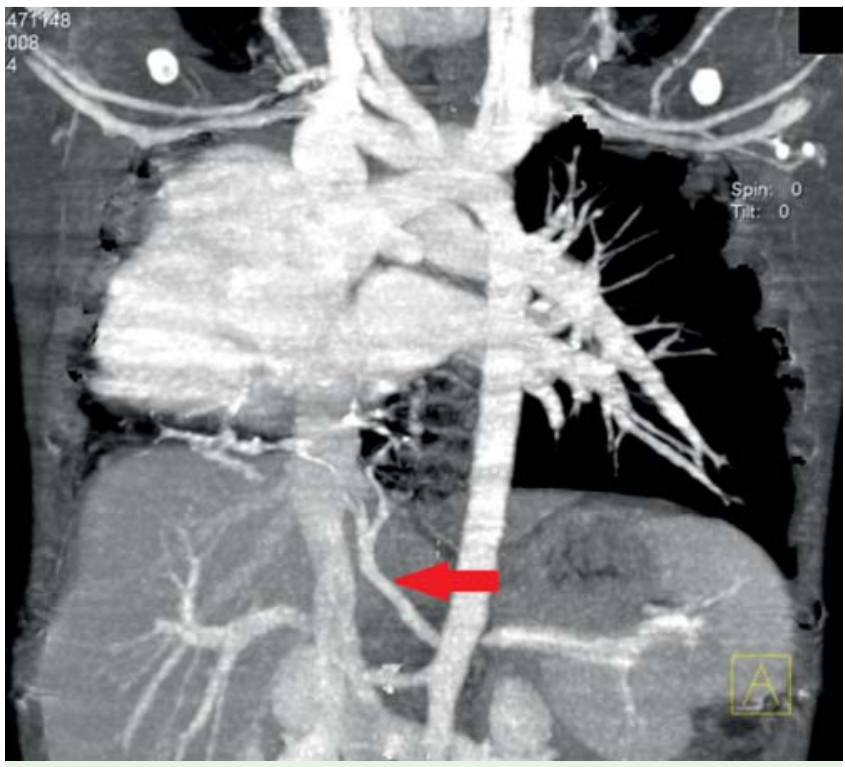

Abb.2 CT-Thorax. Patient (post partum, weiblich): Lungensequester rechts; Pfeil zeigt das den Sequester versorgende Gefäß, das seinen Ursprung aus der Aorta descendens nimmt (Dr. Rompel, Radiologisches Institut, Universitätsklinikum Erlangen [Prof. Dr. med. Michael Uder]).

nahme eines Patienten mit einem post partum diagnostizierten rechtsseitigen Sequester mit deutlicher Volumenverminderung der übrigen rechten Lunge. Aus der Aorta descendens ziehen zwei Gefäße transdiaphragmal nach rechts intrapulmonal mit Abgang in Höhe des Truncus pulmonalis bzw. der Arteria mesenterica superior (siehe Pfeil in $\bullet$ Abb.2). Diese beiden Gefäße verzweigen sich intrapulmonal irregulär und scheinen mit Pulmonalarterienästen zu kommunizieren.

Im Hinblick auf die symptomatische Manifestation herrscht bei den verschiedenen Autoren weitestgehende Übereinstimmung: Neben selten asymptomatischen Verläufen leiden Betroffene zumeist bereits im Säuglingsalter an Beschwerden wie Hustenanfällen, Zyanoseattacken, rezidivierenden pulmonalen Infektionen und einer häufigen Leistungsminderung [13,16 - 19]. Bei den in Erlangen behandelten Patienten konnten diese Angaben bestätigt werden. Bei 21,4\% verlief der Sequester asymptomatisch, während alle anderen Kinder mindestens an einer respiratorischen Auffälligkeit litten. 14,3\% der Betroffenen zeigten eine Dyspnoe, und $28,6 \%$ fielen durch rezidivierende pulmonale Infektionen bzw. Pneumonien auf. Außerdem wurde bei 14,3\% der Kinder eine Leistungsminderung und bei 7,1\% eine Zyanose nachgewiesen.

Diagnostisch wird relativ einheitlich die Röntgenuntersuchung des Thorax als Mittel der ersten Wahl beschrieben. In letzter Zeit werden jedoch zunehmend die Magnetresonanztomografie, die Computertomografie und der Ultraschall zur Identifikation des Sequesters herangezogen, die Darstellung der Gefäßversorgung erfolgt durch Kontrastmittelgabe (CT) oder mit einer Angiografie [12,14]. In unserem Patientenkollektiv dominierte mit 42,9\% ebenfalls die Röntgenuntersuchung des Thorax. Bei 14,3\% der Kinder wurde eine Ultraschalluntersuchung und bei je 7,1\% eine Computertomografie und/oder eine Bronchoskopie durchgeführt. Die Gefäßversorgung wurde in 78,6\% der Fälle mithilfe der Angiografie untersucht, was ebenfalls den literarisch empfohlenen diagnostischen Maßnahmen entspricht. 
In therapeutischer Hinsicht stellt die chirurgische Resektion des Sequesters bzw., bei ausgeprägtem Befund, die Lobektomie die bevorzugte Intervention dar. Nur selten wird die Embolisation der zuführenden Arterie als Maßnahme empfohlen [8,11,20]. Diese Angaben konnten bei dem für diese Arbeit untersuchten Kollektiv nicht bestätigt werden. Bei 35,7\% der Kinder entschieden sich die Chirurgen, die zuführende Sequesterarterie interventionell zu verschließen. Bei ebenfalls 35,7\% wurde der Sequester chirurgisch reseziert. Bei den restlichen Kindern (28,6\%) war keine Intervention nötig.

Lungensequestrationen, v.a. extralobäre, sind häufig mit anderen Fehlbildungen assoziiert. Neben Zwerchfelldefekten, Lungenlappenagenesien oder auch einem Scimitar-Syndrom finden sich bei vielen Patienten kongenitale Herzmissbildungen. Zimmermann et al. [21] gehen in 12\% aller Sequestrationen von Begleitmissbildungen aus. Dabei dominieren Fehlbildungen des Zwerchfells und der Speiseröhre; seltener hingegen finden sich zusätzliche Anomalien der Wirbelsäule oder auch kardiovaskuläre Malformationen. Auch eine Assoziation mit einer Lungenhypoplasie bzw. mit Thoraxdeformitäten sind beobachtet worden. Extralobäre Sequestrationen sind dabei häufiger mit weiteren Fehlbildungen kombiniert als die intralobären Formen $[8,14$, 22 . Mit $71,4 \%$ trat der Sequester bei den für diese Arbeit untersuchten Kindern am häufigsten assoziiert mit angeborenen Herzfehlern auf. Die Hälfte der Patienten wies andere Lungenfehlbildungen und ein Kind (7,1\%) eine zusätzliche Trachealfehlbildung auf. Bei 35,7\% der Betroffenen konnte zusätzlich ein ScimitarSyndrom diagnostiziert werden. 35,7\% litten an diversen anderen Anomalien, wie beispielsweise einem vesikourethralen Reflux oder einer Analatresie. Dies bestätigt die literarische Hypothese, dass Lungensequester meist in Assoziation mit anderen Fehlbildungen und seltener isoliert auftreten.

\section{Lungenzysten}

$\nabla$

Kongenitale zystische Lungenfehlbildungen treten mit einer geschätzten Inzidenz von 2,2\% bei Neugeborenen auf [23]. In der Literatur herrscht eine uneinheitliche Meinung bezüglich der Geschlechterverteilung. Es wird sowohl von einer Dominanz des männlichen als auch des weiblichen Geschlechts berichtet [2326]. Bei dem in Erlangen untersuchten Patientenkollektiv zeigten sich zystische Malformationen mit 83,3\% bevorzugt beim männlichen Geschlecht.

Auch in Bezug auf die Lokalisation der Zysten finden sich in der Literatur unterschiedliche Angaben. Während diverse Autoren von einer bevorzugten Manifestation in einem der beiden Lungenunterlappen ohne Seitendominanz sprechen [24,27-29], gehen andere von einer zufälligen Verteilung aus [26,30,31]. Auch bei der kongenitalen zystisch-adenomatoiden Malformation (CCAM) wird in der Literatur keine Seitendominanz beschrieben, jedoch die bevorzugte Lokalisation in nur einem Lappen [30,32, 33]. Bei den zwölf Erlanger Patienten befanden sich die Zysten bei $33,3 \%$ im linken und bei 16,7\% im rechten Lungenunterlappen. In 33,3\% der Fälle konnten die Raumforderungen im linken Oberlappen nachgewiesen werden. Bei $16,7 \%$ fanden sich multiple bilaterale Zysten. Insgesamt dominierte aber die linke Seite als Prädilektionsort. Abb. 3 zeigt ein Thorax-CT eines männlichen Patienten mit beidseitigen Lungenzysten. Es besteht eine deutliche Rarefizierung der Lungengefäß- und -gerüstzeichnung mit großen Bullae in den Ober- und Unterlappen beidseits, jedoch unterlappenbetont.

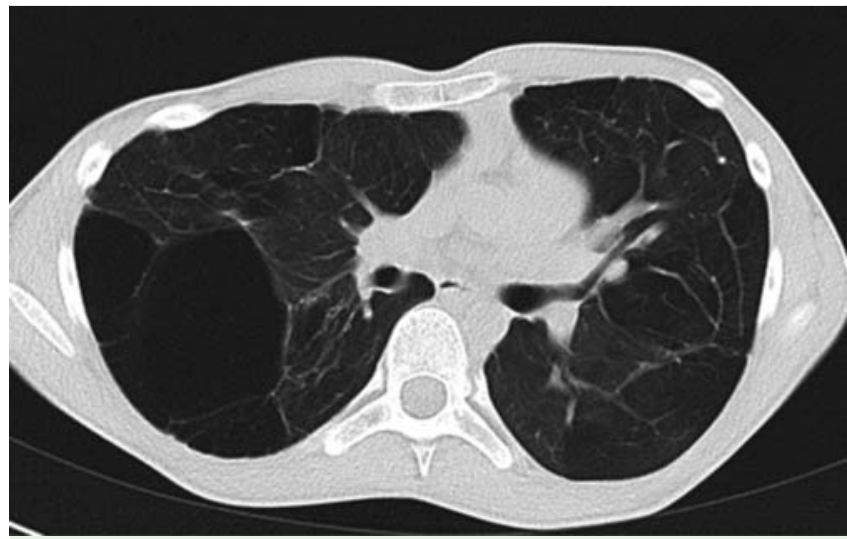

Abb. 3 CT-Thorax. Patient, 5 Monate, männlich: Zystenlunge beidseits (Dr. Rompel, Radiologisches Institut, Universitätsklinikum Erlangen [Prof. Dr. med. Michael Uder]).

Es ist nicht zweifelsfrei nachgewiesen, ob es sich bei den neun in der Kinderklinik Erlangen diagnostizierten CCAM-Fällen tatsächlich um diese Form der Malformation oder um reine Lungenzysten handelte. Die Diagnose ist vom jeweiligen behandelnden Arzt und dessen Einschätzung und Codierung abhängig. Lediglich bei 2 Patienten konnte die Fehlbildung anhand einer pathohistologischen Untersuchung als eine CCAM Typ II bzw. eine CCAM Typ I zweifelsfrei klassifiziert werden.

Klinisch manifestiert sich eine zystische pulmonale Fehlbildung laut literarischen Angaben zumeist in Form von Dyspnoe, Tachypnoe, Zyanoseattacken, einer zystenbedingten trachealen Kompression und rezidivierenden pulmonalen Infektionen. Auch kann es bei ausgeprägten Befunden durch die zum Teil enorme Raumforderung zu einer Mediastinalverschiebung, einer Kompression des Ösophagus oder auch des Herzvorhofes kommen. Es wird aber ebenso von asymptomatischen Verläufen berichtet [23, 34-36]. Auch bei den für diese Arbeit untersuchten Patienten wurde die Hälfte der Kinder aufgrund einer Zyanose und Dyspnoe in Erlangen vorstellig. Rezidivierende pulmonale Infektionen ließen sich bei einem Patienten nachweisen.

Diagnostisch bevorzugen Pädiater neben einer Röntgenuntersuchung des Thorax die Computertomografie, die Magnetresonanztomografie oder auch die Sonografie, in welcher sich die Zysten zumeist als scharf begrenzte, runde Herdschatten darstellen lassen. Nur etwa $10 \%$ der Zysten entgehen der radiologischen Aufnahme $[26,33,37,38]$. Auch bei den in Erlangen therapierten Kindern wurde bei allen 12 Patienten eine Röntgenaufnahme des Thorax durchgeführt. Darüber hinaus konnte die zystische Fehlbildung in einer Ultraschalluntersuchung nachgewiesen werden. Bei mehr als 50\% lieferte zudem eine Computertomografie den Nachweis der Malformation.

Therapeutisch wird in der Literatur die offene chirurgische Resektion der Zyste als Mittel der ersten Wahl beschrieben. Einzelne Zysten werden isoliert chirurgisch entfernt, wohingegen bei multiplen Zysten häufig eine Lappenresektion nötig ist. Uneinigkeit herrscht jedoch darüber, ob asymptomatisch verlaufende Zysten ebenfalls operiert werden sollten oder ob ein abwartendes Vorgehen zu bevorzugen ist $[16,24,39]$. Bei den in dieser Arbeit aufgeführten Fällen wurden 25\% der Kinder lobektomiert. Bei 8,3\% wurde eine Lungenlappenteilresektion vorgenommen. Bei den restlichen Patienten (66,7\%) wurde ein abwartendes Management gewählt. 
In der Literatur finden sich keine eindeutigen Angaben zu weiteren Fehlanlagen, die häufig in Assoziation mit zystischen Lungenfehlbildungen auftreten. Bei den 12 für diese Arbeit untersuchten Kindern wiesen allerdings annähernd die Hälfte zusätzliche kardiale Anomalien auf. Es kann daher davon ausgegangen werden, dass die zystischen Fehlbildungen, ebenso wie viele andere Lungen- bzw. Atemwegsmalformationen, häufig in Kombination mit angeborenen Herzfehlern manifest werden. Lediglich bei der kongenitalen Lymphangiektasie, die ebenfalls zu der Gruppe zystischer Anomalien zu zählen ist, wird eine mögliche Assoziation zu einer totalen Lungenvenenfehleinmündung, einem NoonanSyndrom, einem Hydrops fetalis oder auch einem Down-Syndrom beschrieben $[28,40,41]$. Ein Patient, der laut Krankenakte eine bilaterale angeborene Lymphangiektasie aufwies, litt zusätzlich an einer Trichterbrust.

\section{Lungenhypoplasie}

Die primäre, kongenitale Lungenhypoplasie tritt sehr viel seltener auf als die sekundäre Form $[42,43]$. Sie findet sich bei etwa $10 \%$ aller Neugeborenenautopsien, wobei in mehr als $85 \%$ weitere signifikante Anomalien bestehen $[44,45]$. In Bezug auf eine mögliche Geschlechterverteilung wurden in der Literatur keine Angaben gefunden. Bei den für diese Arbeit untersuchten $36 \mathrm{~Pa}-$ tienten zeigte sich mit 66,7\% zu 33,3\% eine Dominanz des männlichen Geschlechts.

Die sekundäre Lungenhypoplasie entsteht nicht selten aufgrund eines angeborenen Zwerchfelldefektes mit daraus resultierendem Enterothorax, einer Thoraxanomalie, eines Oligohydramnions oder auch einer verminderten fetalen Lungendurchblutung aufgrund eines Herzfehlers. Auch die Pottersequenz kann ursächlich sein $[34,46,47]$, da durch das Oligohydramnion die fruchtwasserabhängige Lungenreifung während der Schwangerschaft gehemmt wird. Diese Angaben können durch die in Erlangen behandelten Kinder bestätigt werden. 19,4\% der Patienten wiesen eine primäre Lungenhypoplasie auf, während es sich bei $80,6 \%$ um eine sekundäre Form handelte. Dabei dominierte mit fast 50\% der angeborene Zwerchfelldefekt als Ursache. In 19,4\% war ein Oligohydramnion, in 5,6\% ein Scimitar-Syndrom und in ebenfalls 5,6\% ein Lungensequester für die Hypoplasie verantwortlich. Jeweils ein Patient $(2,8 \%)$ zeigte die Anomalie aufgrund eines intrauterinen Hydrothorax bzw. als Folge beidseitiger Pleuraergüsse.

Eine Lungenhypoplasie manifestiert sich klinisch zumeist in Form von Atemnot, Zyanoseattacken, Tachydyspnoen und dem möglichen Auftreten eines Pneumothorax. Bei ausgeprägtem Befund versterben die Kinder nicht selten aufgrund einer progressiven Hypoxämie. Bei milden Formen wird aber auch von asymptomatischen Verläufen berichtet $[44,48,49]$. Auch diese Angaben können durch die für diese Arbeit untersuchten Patienten bestätigt werden. Mit 47,2\% dominierte eine respiratorische Insuffizienz, die zumeist unmittelbar postnatal einsetzte. 33,3\% der Kinder litten außerdem an einer starken Zyanose und bei $25 \%$ konnte ein auskultatorisch abgeschwächtes Atemgeräusch festgestellt werden. Jeweils 13,9\% zeigten rezidivierende Sättigungsabfälle und einen reduzierten Allgemeinzustand, während 8,3\% der Patienten durch eine Dyspnoe und Tachypnoe auffällig wurden. Auch in Bezug auf die Letalität bestätigt der hohe Prozentsatz von 44,4\% unter den Erlanger Kindern, dass die Lungenhypoplasie nicht selten zum Tod des Betroffenen führt.

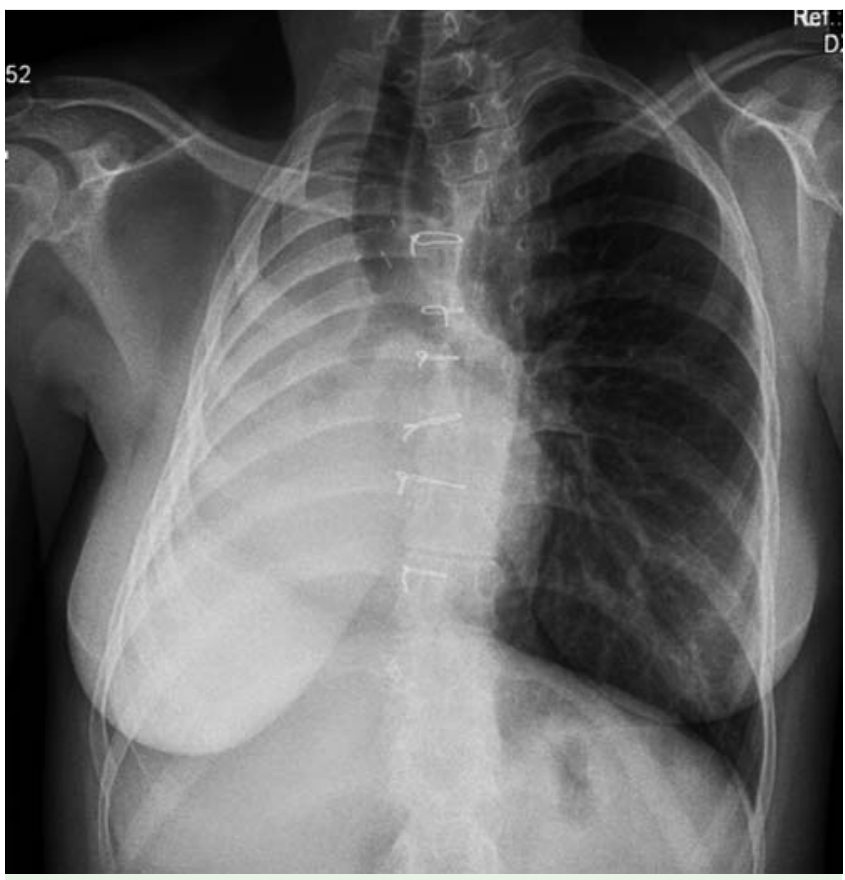

Abb.4 Röntgen-Thorax. Patientin, 15 Jahre, weiblich: Lungenhypoplasie (Dr. Rompel, Radiologisches Institut, Universitätsklinikum Erlangen [Prof. Dr. med. Michael Uder]).

Diagnostisch wird im klinischen Alltag überwiegend die Durchführung einer Röntgenuntersuchung des Thorax empfohlen. Hierbei kann jedoch die Differenzialdiagnose zu einer pulmonalen Agenesie schwierig sein $[6,49]$. Auch bei dem für diese Arbeit untersuchten Patientenkollektiv wurde bei $75 \%$ der Kinder eine Röntgenuntersuchung des Thorax durchgeführt. 69,4\% erhielten eine sonografische Untersuchung und 8,2\% eine Bronchoskopie.

- Abb.4 zeigt eine Thorax-Röntgenaufnahme einer 15 Jahre alten Patientin mit deutlicher Lungenhypoplasie, die sich im Sinne einer Totalverschattung des rechten Hemithorax darstellt. Neben konservativen Maßnahmen kann therapeutisch eine mechanische Ventilation und Oxygenierung sinnvoll sein, um den Gasaustausch zu unterstützen. Spezifische Therapien, um die assoziierte pulmonale Hypertension zu kontrollieren, wie beispielsweise die Inhalation von Stickstoffmonoxid, können sich ebenfalls als hilfreich erweisen. Bei begleitender thorakaler Dystrophie können Rippenexpander das Überleben der Patienten verbessern [7,34,50-52]. Die 36 in Erlangen behandelten Kinder wurden überwiegend konservativ therapiert. Bei zwei Kindern (5,6\%) wurde eine operative Stabilisierung der Thoraxwand bei bestehenden Skelettfehlbildungen vorgenommen. Ein Patient $(2,8 \%)$ wurde aufgrund eines zusätzlich nachgewiesenen Lungensequesters pneumektomiert, während ein weiteres Kind (2,8\%) eine Lungenlappenteilresektion (Wedgeresektion) erhielt.

Die angeborene pulmonale Hypoplasie tritt selten isoliert auf. Es finden sich zahlreiche assoziierte Fehlbildungen. Neben den bereits beschriebenen Zwerchfellhernien finden sich in vielen Fällen Ösophagusatresien und Fehlbildungen der Wirbelsäule, des Skelettsystems, des Urogenitalsystems und kongenitale Herzfehler. Auch weitere Lungenanomalien, wie beispielsweise Lungensequester, werden beobachtet. Die Lungenhypoplasie ist zudem nicht selten mit einer Trisomie 13, 18, 21 oder auch einem Fryns-Syndrom assoziiert $[7,48]$. Auch die 36 in Erlangen vorgefundenen Lungenhypoplasien waren mit anderen angeborenen Anomalien kombiniert. Während bei 38,9\% der Betroffenen zu- 
sätzlich mindestens ein kongenitaler Herzfehler diagnostiziert wurde, wiesen 18,4\% der Kinder weitere Anomalien der Lunge und der pulmonalen Gefäße auf. 11,1\% zeigten Skelettanomalien. Die Tatsache, dass 83,3\% an weiteren Fehlbildungen des Gastrointestinaltraktes oder auch des Urogenitalsystems litten, bestätigt die in der Literatur vorgefundenen Informationen, dass eine Lungenhypoplasie sehr häufig mit anderen Anomalien vergesellschaftet ist.

\section{Primäre Ziliäre Dyskinesie}

Die Inzidenz der Primären Ziliären Dyskinesie (PCD) wird auf etwa 1:10.000-60.000 geschätzt. Bezüglich einer Geschlechterprävalenz gibt es keine genauen Angaben.

In etwa 50\% findet man neben der Ziliendyskinesie außerdem die klassische Kartagener Trias, bestehend aus einem Situs inversus, Bronchitiden und Sinusitiden [53-56].

In Erlangen wurden in den Jahren 2000 bis 2011 drei Kinder aufgrund einer PCD therapiert. Nur ein Patient wies das KartagenerSyndrom auf. Mit 66,7\% dominierte auch hier wieder das männliche gegenüber dem weiblichen Geschlecht.

Symptomatisch manifestiert sich die PCD bereits ab dem Säuglingsalter in Form von rezidivierenden pulmonalen Infektionen. Auch Otitiden, eine eventuelle Schwerhörigkeit, ein chronischer, zumeist produktiver Husten und generalisierte Bronchiektasen sind häufige klinische Zeichen. Es können verschiedenste zilientragende Organe betroffen sein, was zu einer erheblichen klinischen Heterogenität dieser Fehlbildung führt. Die unterschiedlichen Verläufe resultieren außerdem aus den verschiedenen genetischen Defekten und exogenen Faktoren, die die Symptomatik beeinflussen [55-58]. Die in Erlangen untersuchten Patienten bestätigen diese Literaturangaben. Alle drei Kinder litten bereits ab dem Säuglingsalter an rezidivierenden Rhinitiden, Sinusitiden, Bronchitiden und Pneumonien. Aufgrund der Sekretretention zeigten die Kinder außerdem eine angestrengte Respiration mit auskultatorisch wahrnehmbaren Rasselgeräuschen, einem verschärften Exspirium und einem exspiratorischen Giemen. Zusätzlich manifestierte sich die PCD in Form von rezidivierenden Hustenanfällen mit eitrigem Auswurf.

Diagnostisch kann diese Fehlbildung mit einem Abstrich der Nasenschleimhaut oder anhand einer bronchoskopisch gewonnenen Schleimhautprobe elektronenmikroskopisch nachgewiesen werden. Eine genetische Untersuchung kann die zugrunde liegende Mutation in den bisher identifizierten Genen häufig bestätigen $[57,59,60]$. Manche Autoren empfehlen eine Darstellung der Lungen mit MRT oder CT, um eventuell bestehende sekundäre Lungenveränderungen wie Bronchiektasen darzustellen $[61,62]$. Auch bei den drei Erlanger Patienten konnte der Verdacht einer PCD durch eine mikroskopische Zilienfunktionsuntersuchung bestätigt werden. Zudem wurde bei $66,7 \%$ eine Röntgenuntersuchung des Thorax durchgeführt, die bei einem Kind zusätzlich die Diagnose eines Situs inversus erbrachte. Jeweils 33,3\% der Patienten erhielten eine Bronchoskopie, eine Lungenfunktionsuntersuchung, einen thorakalen Ultraschall und eine HNO-Untersuchung.

Eine spezifische Therapie existiert derzeit nicht. Das Hauptziel besteht in der Vermeidung möglicher Komplikationen. Neben antibiotischen, inhalativen oder physiotherapeutischen Maßnahmen stellen auch operative Interventionen therapeutische Ansätze dar. Bei suffizienter, frühzeitig einsetzender Behandlung ist die Prognose als gut einzuschätzen $[53,57,63,64]$. Auch bei den Erlanger Patienten wurden die rezidivierenden pulmonalen Infektionen mithilfe von Antibiotika therapiert. Außerdem wurden den Eltern physiotherapeutische Maßnahmen und Inhalationen empfohlen.

Eine Reihe unterschiedlicher Störungen wie Herzfehler, ein Hydrocephalus internus, zystische Nierenerkrankungen oder auch eine Schwerhörigkeit können mit der PCD assoziiert auftreten und das Erkrankungsbild verkomplizieren [56,57,65]. Bei den für diese Arbeit analysierten Patienten wies nur ein Kind zusätzliche Herzfehler in Form einer D-Transposition der großen Arterien, einer Trikuspidalklappen- bzw. Aortenklappeninsuffizienz und einer Dextrokardie aufgrund des bestehenden Situs inversus auf. Zudem litt das Kind an einem angeborenen gastroösophagealen Reflux. Bei den beiden anderen Patienten konnten keine weiteren Fehlbildungen diagnostiziert werden.

\section{Tracheomalazien und Trachealstenosen}

\section{$\nabla$}

Diese beiden Anomalien werden in der Literatur als seltene Fehlbildungen beschrieben. Die Inzidenz der Tracheomalazie beträgt etwa $0,001 \%-1,5 \%$, während die primäre Trachealstenose bei ca. 1:50.000 Neugeborenen diagnostiziert wird [66,67]. In Erlangen wurden 38 Kinder aufgrund mindestens einer der beiden Anomalien behandelt. Dabei lag sowohl bei den Tracheomalazien als auch bei der angeborenen Trachealstenose eine deutliche Bevorzugung des männlichen Geschlechts vor.

Bei beiden Fehlbildungsformen unterscheidet man primäre, kongenitale von den häufiger auftretenden, sekundären Formen [7, 34,68]. Diese Angaben können durch die 38 Kinder der Erlanger Universitätsklinik bestätigt werden. Die primäre Tracheomalazie konnte nur bei insgesamt 15,4\% der Kinder und die primäre kongenitale Trachealstenose in 9,5\% der Fälle diagnostiziert werden. Bei den restlichen Patienten bestanden sekundäre Formen, beispielsweise als Folge einer trachealen Kompression von außen durch einen abnormen Gefäßverlauf.

Die Klinik resultiert bei der Tracheomalazie aus der unzureichenden Stabilität der Luftröhre und der dadurch bedingten funktionellen Stenose. Die Kinder leiden neben Zyanoseattacken und Dyspnoeanfällen häufig an einem Stridor. Außerdem kann es zu Komplikationen bei der Nahrungsaufnahme kommen. Auch Asphyxien und Tachypnoen werden häufig beobachtet [51,56, $66,69]$. Diese Symptome traten auch bei den Erlanger Patienten auf. Mit 55,3\% dominierte ein auskultatorisch deutlich wahrnehmbarer inspiratorischer Stridor. Darüber hinaus fielen die Kinder durch Zyanose- und Apnoeanfälle, eine Tachydyspnoe, eine respiratorische Insuffizienz und in 7,9\% durch rezidivierende pulmonale Infekte auf.

Diagnostisch werden im klinischen Alltag neben bronchoskopischen bzw. tracheoskopischen Untersuchungen die Röntgenaufnahme des Thorax, die Magnetresonanztomografie und die Computertomografie angewandt. Auch die Lungenfunktion kann wichtige Hinweise liefern [7,34, 70]. In der Kinderklinik Erlangen erhielten 55,3\% der Kinder Röntgenaufnahmen des Thorax, 52,6\% eine Broncho- bzw. Tracheoskopie und 34,2\% eine Computertomografie. Bei $26,3 \%$ wurde eine Ultraschalluntersuchung und bei 13,2\% ein MRT der Halsweichteile durchgeführt. Eine Messung der Lungenfunktion erfolgte bei 7,9\% der Kinder.

Therapeutisch existiert derzeit keine einheitliche Leitlinie. Während einige Autoren davon ausgehen, dass vor allem die milderen Ausprägungsformen durch das tracheale Wachstum im Laufe der Kindheit an Bedeutung verlieren und somit ein konservatives 
Management bevorzugt werden sollte [71], müssen vor allem bei klinisch manifesten Malazien bzw. Stenosen auch Segmentresektionen, Trachealplastiken oder Stenteinlagen durchgeführt werden $[6,7,72]$.

In Erlangen wurden 7,9\% primär durch ein Tracheostoma versorgt. Eine operative Intervention im Sinne einer Trachealerweiterung, einer Resektion des betroffenen Tracheaabschnittes bzw. eines trachealen Stentings wurde bei 15,8\% durchgeführt. Bei $15,8 \%$ der Patienten wurde eine Korrektur der bestehenden Gefäßmissbildung vorgenommen. Die restlichen Kinder wurden konservativ therapiert. Somit decken sich die in der Literatur gefundenen Angaben mit den in den Krankenakten vorgefundenen Informationen.

Sowohl die Tracheomalazie als auch die Trachealstenose treten häufig in Assoziation mit weiteren Fehlbildungen auf. Während sowohl Harrison et al., Nelson et al. als auch andere Arbeitsgruppen von einem gehäuften kombinierten Auftreten einer Trachealstenose mit einer Lungenagenesie bzw. bronchialen Anomalie berichten $[73,74]$, finden sich bei beiden kongenitalen Lungenfehlbildungen außerdem zusätzlich nicht selten tracheoösophageale Fisteln, Larynxspalten und Bindegewebserkrankungen [6, $7,34,67]$. Mit 44,7\% wies in Erlangen fast die Hälfte der betroffenen Kinder neben einer Tracheomalazie bzw. -stenose einen zusätzlichen kongenitalen Herzfehler auf. Bei 21,1\% bestanden außerdem weitere Anomalien des Lungenparenchyms bzw. der pulmonalen Gefäße. 15,8\% der Patienten litten an trachealen Fehlbildungen, und 10,5\% zeigten verschiedene Formen an Skelettfehlbildungen. Bei 63,2\% bestanden darüber hinaus weitere Anomalien, beispielsweise des Gastrointestinal- bzw. Urogenitaltraktes. Auf eine häufige Assoziation mit einem zusätzlichen Herzfehler wurde in der Literatur nicht explizit hingewiesen.

\section{Alveolokapilläre Dysplasie}

Diese angeborene Anomalie tritt sehr selten auf. In Bezug auf die Geschlechterverteilung scheinen Jungen und Mädchen gleich häufig betroffen zu sein $[75,76]$. Dies konnte durch die Erlanger Patienten bestätigt werden. In den 11 Jahren wurden zwei Kinder aufgrund einer alveolokapillären Dysplasie behandelt. Dabei war ein Patient weiblichen und ein Patient männlichen Geschlechts.

Klinisch zeichnet sich diese Fehlbildung hauptsächlich durch eine ausgeprägte pulmonale Hypertension und eine starke Atemnot aus. Darüber hinaus kann sich ein Pneumothorax ausbilden [77, 78]. Auch die beiden Erlanger Kinder wiesen unmittelbar postnatal eine respiratorische Insuffizienz, eine pulmonale Hypertonie und eine deutliche Zyanose auf.

Zur Klärung der bestehenden Atemnot wird häufig eine Röntgenaufnahme des Thorax angefertigt. Die pulmonale Hypertension wird mit einer Echokardiografie und einer Angiokardiografie abgeklärt. Mit der feingeweblichen Untersuchung des Lungengewebes kann die alveolokapilläre Dysplasie nachgewiesen werden. $[34,79]$. In den Erlanger Fällen lieferte eine thorakale Röntgenaufnahme unspezifische Hinweise auf eine alveolokapilläre Dysplasie. Eine Echokardiografie zeigte außerdem den Befund einer bestehenden pulmonalen Hypertension. Eine postmortal durchgeführte histopathologische Untersuchung der Lunge bestätigte letztendlich die Diagnose.

Eine therapeutische Leitlinie existiert derzeit nicht. Die meisten der betroffenen Kinder versterben innerhalb der ersten Lebenstage [76, 80]. Der Versuch einer extrakorporalen Membranoxygenierung oder auch der Einsatz von Prostazyklin oder Stickstoff- monoxid können das Überleben der Patienten nur unwesentlich verlängern $[78,81]$. Auch die beiden Kinder in Erlangen verstarben unmittelbar im Neugeborenenalter.

Die alveolokapilläre Dysplasie tritt nur selten isoliert auf. Die Fehlbildung findet sich häufig assoziiert mit kardiovaskulären, gastrointestinalen, urogenitalen und muskuloskelettalen Anomalien. Auch Lungenlappungsanomalien oder weitere Fehlbildungen der Lungengefäße treten auf [82-84]. Auch die beiden für diese Arbeit untersuchten Kinder wiesen weitere Fehlbildungen auf. Neben einer Lungenhypoplasie, diversen Herzanomalien und einer Nierendysplasie war die alveolokapilläre Dysplasie außerdem mit einem Pancreas anulare und einer Duodenalatresie assoziiert.

\section{Agenesie der Lunge \\ $\nabla$}

Die Inzidenz der Lungenagenesie ist nicht gesichert. Schmidt et al. gehen von einer Häufigkeit von 1:10.000 bis 1:15.000 bei allen durchgeführten Autopsien aus [27,85,86]. Die einseitige Lungenagenesie dominiert dabei gegenüber der bilateralen Form [45]. Schechter et al. und Kumar et al. beschreiben eine leichte Bevorzugung des weiblichen Geschlechts (1,3:1) [86,87]. Die Prognose einer linksseitigen Agenesie scheint deutlich besser zu sein als die einer rechtsseitigen. Begründet ist dies durch die Tatsache, dass die rechtsseitige Lungenagenesie häufiger mit kardiovaskulären Anomalien einhergeht $[7,88]$.

In den Jahren von 2000 bis 2011 wurde in Erlangen lediglich ein Patient aufgrund einer linksseitigen Lungenagenesie behandelt. Symptomatisch fallen viele der Patienten unmittelbar postnatal durch eine Ateminsuffizienz, ein einseitig fehlendes Atemgeräusch, ein Atemnotsyndrom oder rezidivierende pulmonale Infektionen bzw. Bronchitiden auf. Darüber hinaus zeigen die Kinder häufig eine Zyanose, eine Dyspnoe und Tachypnoen. Im späteren Verlauf kann es, bedingt durch das fehlende Lungenparenchym, zu einer Thoraxasymmetrie der betroffenen Seite kommen. Selten verläuft die unilaterale Agenesie auch asymptomatisch, da die andere Lunge kompensatorisch hypertrophiert ist und mehr leistet $[89,90]$. Der in Erlangen therapierte Patient wies ebenfalls unmittelbar postnatal Anzeichen einer Zyanose und eine Tachydyspnoe auf. Außerdem war auskultatorisch ein intermittierender inspiratorischer Stridor, ein exspiratorisches Giemen und ein linksseitig fehlendes Atemgeräusch zu vernehmen. Postprandial zeigte das Kind eine Schluckstörung, die sich bei respiratorischer Anstrengung verschlimmerte.

Diagnostisch wird die Röntgenuntersuchung bevorzugt, wobei typischerweise eine homogene Verschattung auf der betroffenen Seite auffällt. Auch die Computertomografie des Thorax ist zum Nachweis einer Agenesie hilfreich. Ebenso sollte die Durchführung einer Bronchoskopie in Betracht gezogen werden. Hierbei kann unter Umständen ein Fehlen der Carina bzw. des jeweiligen Hauptbronchus nachgewiesen werden $[86,88,91]$. Die in der Literatur empfohlenen diagnostischen Maßnahmen wurden auch bei dem für diese Arbeit untersuchten Patienten angewandt. Neben Röntgenaufnahmen des Thorax, einer Röntgenbreischluckuntersuchung, einer Bronchoskopie, einer Tracheoskopie bzw. -grafie wurden außerdem computertomografische Aufnahmen durchgeführt. Darüber hinaus wurden Echokardiografien, Elektrokardiografien und eine Chromosomenanalyse zum Ausschluss eines eventuell bestehenden Syndroms angeordnet. 


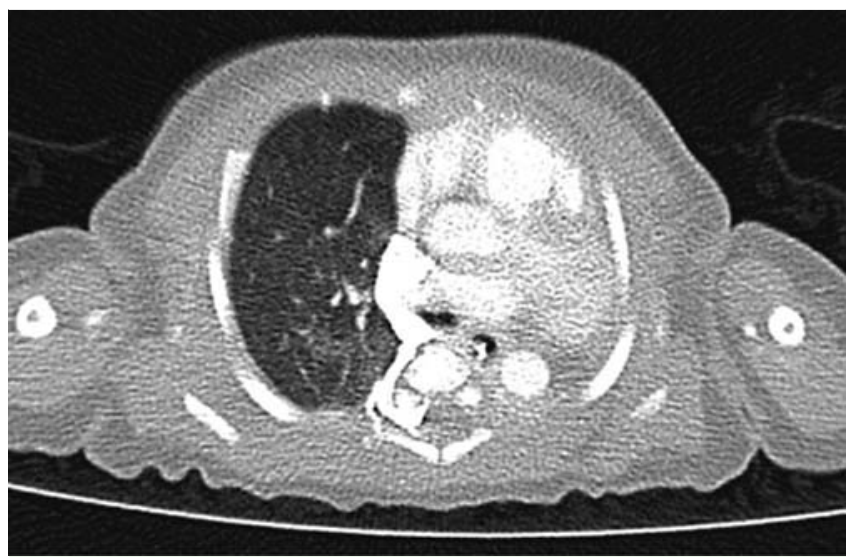

Abb.5 CT-Thorax. Patient, post partum, männlich: Agenesie der linken Lunge (Dr. Rompel, Radiologisches Institut, Universitätsklinikum Erlangen [Prof. Dr. med. Michael Uder]).

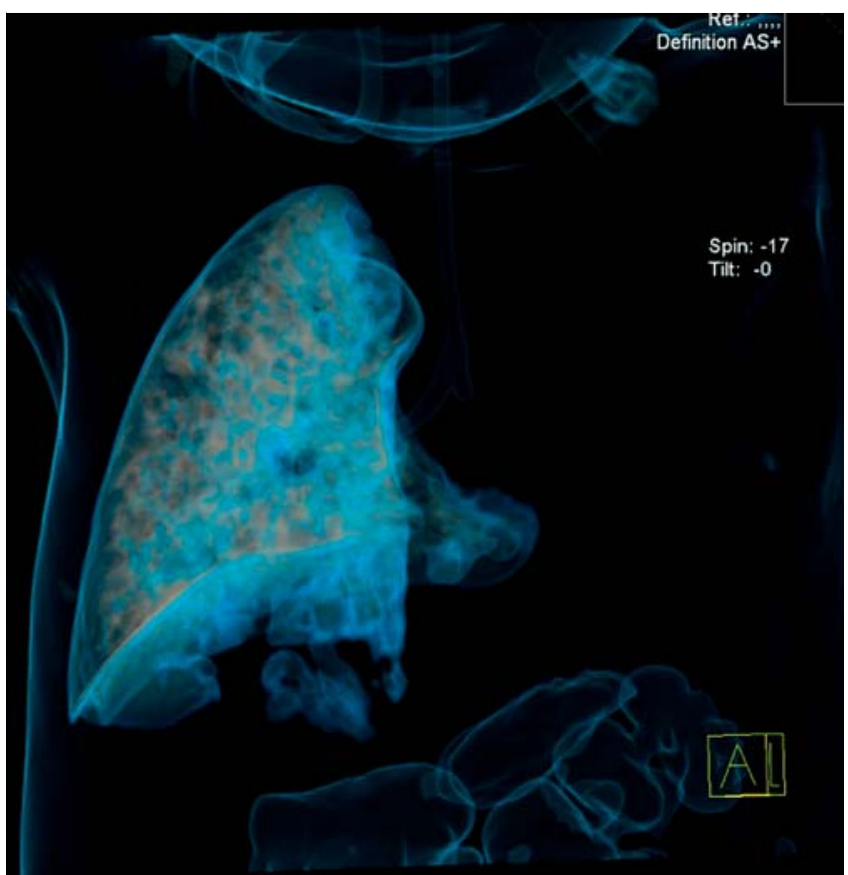

Abb. 6 Patient, post partum, männlich: Agenesie der linken Lunge (Dr. Rompel, Radiologisches Institut, Universitätsklinikum Erlangen [Prof. Dr. med. Michael Uder]).

Die Abb. 5 und $\bullet$ Abb. 6 zeigen Thoraxaufnahmen des Patienten mit einer kompletten Verschattung des linken Hemithorax bei bekannter Lungenagenesie links. Dabei dehnt sich der rechte Lungenflügel ventral bis auf die Gegenseite aus. Das Mediastinum hat sich aufgrund der Agenesie nach links verlagert. Außerdem ist das Zwerchfell links, bedingt durch die Fehlanlage, bei den posterior-anterior-Aufnahmen im Gegensatz zur rechten Seite nicht eindeutig abgrenzbar.

Die Therapie beschränkt sich auf supportive Maßnahmen der Physiotherapie, die Korrektur assoziierter Fehlbildungen und die Prävention von Infektionen. Relativ neu ist der Einsatz von Gewebeexpandern zur Minderung der Mediastinalverlagerung und daraus resultierender Komplikationen [34,92]. Bei dem Erlanger Kind wurde die Agenesie konservativ therapiert. Aufgrund der ausgeprägten Trachealstenose erhielt es außerdem ein Tracheostoma und eine plastische Trachealerweiterung.
Die Häufigkeit assoziierter Fehlbildungen wird in der Literatur mit $50 \%$ bis $75 \%$ beschrieben, wobei kardiovaskuläre Anomalien, Fehlbildungen des Skelett- bzw. Urogenitalsystems und solche des Gastrointestinaltraktes dominieren. Wie bereits erwähnt, sind vor allem rechtsseitige Lungenagenesien häufig mit angeborenen Herzfehlern assoziiert [88,93]. Auch bei dem Erlanger Jungen fanden sich diverse andere kongenitale Anomalien. Neben der Lungenagenesie litt das Kind unter einer massiven Tracheomalazie mit einer sekundären Trachealstenose, einem kongenitalen Megaureter und einer Hydronephrose links. Außerdem bestanden Herzfehler in Form eines Atriumseptumsdefekts (ASD) und eines persistierenden Ductus arteriousus Botalli (PDA).

\section{Schlussfolgerung}

\section{$\nabla$}

Die durchgeführte Analyse von Fällen an angeborenen Lungenund Atemwegserkrankungen an der Kinderklinik Erlangen zeigt, dass derartige Fehlbildungen selten sind, aber ein hohes Maß an diagnostischen und therapeutischen Maßnahmen erfordern. Trotz der bereits erzielten Fortschritte in der Diagnostik und Therapie besteht weiterhin erheblicher Forschungsbedarf, um Kindern mit angeborenen Lungen- und Atemwegsfehlbildungen ein normales Leben zu ermöglichen. Die Tatsache, dass derartige Anomalien sehr häufig mit anderen, insbesondere kardiovaskulären Fehlbildungen assoziiert sind, erfordert eine enge interdisziplinäre Zusammenarbeit der verschiedenen klinischen Fachrichtungen.

\section{Interessenkonflikt}

$\nabla$

Die Autoren geben an, dass kein Interessenkonflikt besteht

\section{Literatur}

1 Khalil KG, Kilman JW. Pulmonary sequestration. J Thorac Cardiovasc Surg 1975; 70: 928-937

2 Carter R. Pulmonary sequestration. Ann. Thorac. Surg 1969; 7: 68 -88

3 Baumgartl $F$, Gremmel $H$. Die Lungensequestration und ihre klinische Bedeutung. Thorac Cardiovasc Surg 1959; 7: 547-559

4 Kabnick EM, Adler L, Susin M et al. Pulmonary Sequestration. J Natl Med Assoc 1984; 76: $911-912$

5 Schneider D. Therapie, Diagnostik und Langzeitverlauf bei angeborenen Lungenfehlbildungen an der Klinik für Kinder und Jugendliche der Universität Erlangen-Nürnberg von 1980-1995. Erlangen: Med Diss1998

6 Lentze MJ, Schaub J, Schulte FJ et al., Hrsg. Pädiatrie. Grundlagen und Praxis. Heidelberg: Springer Medizin; 2007

7 Chernick V, Boat TF, Wilmott RW et al., Hrsg. Kendig's disorders of the respiratory tract in children. Philadelphia, PA: Saunders/Elsevier; 2006

8 Barbut J, Fernandez C, Blanc F et al. Pulmonary sequestration of the left upper lobe associated with a bronchogenic cyst: Case report of an exceptional association. Pediatr Pulmonol 2010: 509-511

9 O'Mara CS, Baker RR, Jeyasingham K. Pulmonary sequestration. Surg Gynecol Obstet 1978; 147: 609-616

10 Aryal G, Pathak $V$. Bronchopulmonary sequestration presenting as recurrent pneumonia. WMJ 2011; 110: 240-242

11 Diks J, Schütte PR, Cheung $D$ et al. Treatment of pulmonary sequestrations by means of endovascular embolization: future or fashion? Case Report Med 2011: 1 -3

12 Westphal FL, de Lima LC, Lima Netto JC et al. Tumor carcinoide e sequestro pulmonar. J bras pneumol 2012; 38: $133-137$

13 Thalhammer GH, Eber E, Zach MS. Angeborene Fehlbildungen des unteren Respirationstraktes. Pneumologe 2006; 3: 372 - 380

14 Paquet KJ, Savić B, Kreutzberg B. Zur klinischen Bedeutung der intralobären Lungensequestration. Langenbecks Arch Chiv 1970; 327: $561-567$

15 Mirza B, Saleem M, Ijaz $L$ et al. Pulmonary sequestration cyst in a patient of cerebral palsy. Lung India 2011; 28: 209-211 
16 Konietzko N, Wendel H, Wiesner B, Hrsg. Erkrankungen der Lunge. Berlin; New York: Walter de Gruyter; 1995

17 Collin PP, Desjardins JG, Khan AH. Pulmonary sequestration. J Pediatr Surg 1987; 22: $750-753$

18 Sauvanet A, Regnard JF, Calanducci F et al. Les séquestrations pulmonaires. Aspects chirurgicaux à propos de 61 observations. Rev Pneumol Clin 1991; 47: 126-132

19 Berna $P$, Cazes A, Bagan $P$ et al. Intralobar sequestration in adult patients. Interactive CardioVascular and Thoracic Surgery 2011; 12: 970-972

20 Piccione $W$, Burt ME. Pulmonary sequestration in the neonate. Chest 1990; 97: 244-246

21 Zimmermann Th, Deeg KH, Hümmer HP et al. Die intralobäre Lungensequestration. Mon.schr. Kinderheilk 1986; 134: 869-873

22 Hasche E. Die intralobäre Sequestration. Thorac cardiovasc Surg 1962; 10: $15-32$

23 Makhija Z, Moir CR, Allen MS et al. Surgical Management of Congenital Cystic Lung Malformations in Older Patients. The Annals of Thoracic Surgery 2011; 91: 1568-1573

24 St-Georges $R$, Deslauriers J, Duranceau A et al. Clinical spectrum of bronchogenic cysts of the mediastinum and lung in the adult. Ann Thorac Surg 1991; 52: 6-13

25 Sarper A, Ayten A, Golbasi I et al. Bronchogenic cyst. Tex Heart Inst J 2003; 30: $105-108$

26 Suen HC, Mathisen DJ, Grillo HC et al. Surgical management and radiological characteristics of bronchogenic cysts. Ann Thorac Surg 1993; 55: $476-481$

27 Langhorst H, Mall W. Fehlbildungen der Lunge. Pneumologie 1993; 47: $566-572$

28 Joshi J, Gothi D. A 38-year-old man with lung cysts. Ann Thorac Med 2011; 6: 231

29 Aktoğu S, Yuncu G, Halilçolar H et al. Bronchogenic cysts: clinicopathological presentation and treatment. Eur. Respir. J 1996; 9: 2017-2021

30 Chernick V, Boat TF, Kendig EL, Hrsg. Kendig's Disorders of the respiratory tract in children. Philadelphia: W. B. Saunders Company; 1967

31 Kim WS, Lee KS, Kim IO et al. Congenital cystic adenomatoid malformation of the lung: CT-pathologic correlation. AJR Am J Roentgenol 1997; 168: $47-53$

32 Wolf SA, Hertzler JH, Philippart AI. Cystic adenomatoid dysplasia of the lung. J Pediatr Surg 1980; 15: 925 - 930

33 Chaouachi S, Ben Hamida E, Ben Fraj $N$ et al. Congenital cystic adenomatoid malformation of the lung: two case reports. Tunis Med 2011; 89: $55-58$

34 Rieger C, von der Hardt H, Sennhauser FH et al., Hrsg. Pädiatrische Pneumologie. Berlin; New York: Springer; 1999

35 Reinhardt D, Griese M, Hrsg. Therapie der Krankheiten im Kindes- und Jugendalter. Berlin: Springer Medizin; 2007

36 Rodgers BM, Harman PK, Johnson AM. Bronchopulmonary foregut malformations. The spectrum of anomalies. Ann Surg 1986; 203: 517 - 524

37 Tireli GA, Ozbey H, Temiz A et al. Bronchogenic Cysts: A Rare Congenital Cystic Malformation of the Lung. Surg Today 2004; 34: 573 - 576

38 Beddy P, Babar J, Devaraj A. A practical approach to cystic lung disease on HRCT. Insights Imaging 2011; 2: 1 - 7

39 Kirmani B, Kirmani B, Sogliani F. Should asymptomatic bronchogenic cysts in adults be treated conservatively or with surgery? Interact Cardiovasc Thorac Surg 2010; 11: 649-659

40 Straaten HLM, Gerards LJ, Krediet TG. Chylothorax in the neonatal period. Eur J Pediatr 1993; 152: 2 -5

41 Annam V, Korishetty SI, Yelikar BR et al. Bilateral congenital cystic adenomatoid malformation, stocker type III with associated findings and review of literature. Indian J Pathol Microbiol 2010; 53: 331 - 333

42 Mendelsohn G, Hutchins GM. Primary pulmonary hypoplasia: report of a case with polyhydramnios. Am J Dis Child 1977; 131: 1220-1223

43 Moore KL, Persaud VN, Hrsg. Embryologie - Entwicklungsstadien Frühentwicklung - Organogenese - Klinik. München Jena: Elsevier Urban \& Fischer; 2003

44 Page DN, Stocker JT. Anomalies associated with pulmonary hypoplasia. Am Rev Respir Dis 1982; 125: 216-221

45 Dail DH, Hammar SP, Hrsg. Pulmonary Pathology. New York: Springer; 1998

46 Nakayama DK, Glick PL, Harrison MR et al. Experimental pulmonary hypoplasia due to oligohydramnios and its reversal by relieving thoracic compression. J Pediatr Surg 1983; 18: 347 - 353

47 Perlman $M$, Williams J, Hirsch $M$. Neonatal pulmonary hypoplasia after prolonged leakage of amniotic fluid. Arch Dis Child 1976; 51: 349 - 353
48 Swischuk LE, Richardson CJ, Nichols M et al. Bilateral pulmonary hypoplasia in the neonate. AJR Am J Roentgenol 1979; 133: 1057 - 1063

49 Langer R, Kaufmann HJ. Primary (isolated) bilateral pulmonary hypoplasia: a comparative study of radiologic findings and autopsy results. Pediatr Radiol 1986; 16: 175-179

50 Schwenke de Wall $H$. Kongenitale Lungenfehlbildungen. Eine Analyse von 21 Fällen. Göttingen: Zahnmed. Diss; 1978

51 Kliegman RM, Stanton BF, Schor NF et al., Hrsg. Nelson textbook of pediatrics. Philadelphia: Saunders Elsevier; 2011

52 Thomas RJ, Lathif HC, Sen S et al. Varied presentations of unilateral lung hypoplasia and agenesis: a report of four cases. Pediatric Surgery International 1998; 14: $94-95$

53 Gärtig S, Konietzko N. Primäre ziliare Dyskinesie. Pneumologe 2006; 3: $364-371$

54 Tanaka K, Sutani A, Uchida Y et al. Ciliary ultrastructure in two sisters with Kartagener's syndrome. Med Mol Morphol 2007; 40: 34 - 39

55 Omran H. Genetische Defekte bei primärer ziliärer Dyskinesie. Monatsschr Kinderheilkd 2005; 153: $246-254$

56 Sitzmann FC, Bartmann P, Hrsg. Pädiatrie. Stuttgart: Thieme; 2007

57 Armengot Carceller M, Mata Roig M, Milara Payá X et al. Discinesia ciliar primaria. Ciliopatías. Acta Otorrinolaringol Esp 2010; 61: 149-159

58 Prulière-Escabasse V, Coste A, Chauvin P et al. Otologic features in children with primary ciliary dyskinesia. Arch Otolaryngol Head Neck Surg 2010; 136: $1121-1126$

59 Stannard WA, Chilvers MA, Rutman AR et al. Diagnostic testing of patients suspected of primary ciliary dyskinesia. Am J Respir Crit Care Med 2010; 181: 307 - 314

60 Skeik N, Jabr FI. Kartagener syndrome. Int J Gen Med 2011; 4: 41 - 43

61 Puderbach M, Kauczor HU. Assessment of lung function in children by cross-sectional imaging: techniques and clinical applications. Pediatr Radiol 2006; 36: $192-204$

62 Montella S, Santamaria F, Salvatore M et al. Lung disease assessment in primary ciliary dyskinesia: a comparison between chest high-field magnetic resonance imaging and high-resolution computed tomography findings. Ital J Pediatr 2009; 35: 24

63 McManus IC, Mitchison HM, Chung EMK et al. Primary ciliary dyskinesia (Siewert's/Kartagener's syndrome): respiratory symptoms and psycho-social impact. BMC Pulm Med 2003; $3: 4$

64 Stillwell PC, Wartchow EP, Sagel SD. Primary Ciliary Dyskinesia in Children: A Review for Pediatricians, Allergists, and Pediatric Pulmonologists. Pediat Allergy Immunol Pulmonol 2011; 24: 191 - 196

65 Afzelius B, Mossberg B. Immotile cilia. Thorax 1980; 35: 401 - 404

66 Agarwal A, Mis AK, Gupta SlK et al. High incidence of tracheomalacia in longstanding goiters: experience from an endemic goiter region. World J Surg 2007; 31: 832-837

67 Krause U, Rödel RMW, Paul T. Isolated congenital tracheal stenosis in a preterm newborn. Eur J Pediatr 2011; 170: 1217 - 1221

68 Reiss M, Hrsg. Facharztwissen HNO-Heilkunde. Differenzierte Diagnostik und Therapie. Heidelberg: Springer; 2009

69 Lang FJ, Hurni M, Monnier P. Long-segment congenital tracheal stenosis: treatment by slide-tracheoplasty. J Pediatr Surg 1999; 34: 1216 1222

70 Elliott $M$, Roebuck $D$, Noctor $C$ et al. The management of congenital tracheal stenosis. Int J Pediatr Otorhinolaryngol 2003; 67: 183-192

71 Fenner A, von der Hardt H, Hrsg. Pädiatrische Pneumologie. Berlin; Heidelberg: Springer; 1985

72 Yamada S, Kikuchi K, Kosaka A et al. Surgical management of idiopathic tracheal stenosis. Jpn J Thorac Cardiovasc Surg 2009: 335 - 338

73 Harrison MR, Heldt GP, Brasch RC et al. Resection of distal tracheal stenosis in a baby with agenesis of the lung. J Pediatr Surg 1980; 15: $938-943$

74 Nelson CS, Mc Millan IKR, Bharucha PK. Tracheal stenosis, pulmonary agenesis, and patent ductus arteriosis. Thorax 1967; 22: 7-12

75 Merchak A, Lueder GT, White FV et al. Alveolar capillary dysplasia with misalignment of pulmonary veins and anterior segment dysgenesis of the eye: a report of a new association and review of the literature. J Perinatol 2001; 21: $327-330$

76 Vassal HB, Malone M, Petros AJ et al. Familial persistent pulmonary hypertension of the newborn resulting from misalignment of the pulmonary vessels (congenital alveolar capillary dysplasia). J Med Genet 1998; 35: 58-60

77 Roy PG, Patel P, Vayalakkad A et al. Alveolar capillary dysplasia presenting as pneumothorax: a case report and review of literature. Pediatr Surg Int 2007; 23: 915 -917 
78 Gutierrez C, Rodriguez A, Palenzuela S et al. Congenital Misalignment of Pulmonary Veins with Alveolar Capillary Dysplasia Causing Persistent Neonatal Pulmonary Hypertension: Report of Two Affected Siblings. Pediatric and Developmental Pathology 2000; 3: 271-276

79 Pucci A, Zanini C, Ferrero F et al. Misalignment of lung vessels: diagnostic role of conventional histology and immunohistochemistry. Virchows Arch 2003; 442: 597-600

80 Al-Hathlol K, Phillips S, MKS et al. Alveolar capillary dysplasia. Report of a case of prolonged life without extracorporeal membrane oxygenation (ECMO) and review of the literature. Early Hum Dev 2000; 57: $85-94$

81 Kinugasa $H$, Horigome $H$, Sugiura $M$ et al. Intravenous prostacyclin combined with inhaled nitric oxide therapy for an infant with alveolar capillary dysplasia. Pediatr Int 2002; 44: 525-527

82 Janney CG, Askin FB, Kuhn C. Congenital alveolar capillary dysplasia an unusual cause of respiratory distress in the newborn. Am J Clin Pathol 1981; 76: $722-727$

83 Wagenvoort CA. Misalignment of lung vessels: A syndrome causing persistent neonatal pulmonary hypertension. Human Pathology 1986; 17: $727-730$

84 Roth $W$, Bucsenez D, Bläker $H$ et al. Misalignment of pulmonary vessels with alveolar capillary dysplasia: association with atrioventricular septal defect and quadricuspid pulmonary valve. Virchows Arch 2006; 448: $375-378$
85 Schmidt ME, Preston A, Bellah RD. The "pseudo-ring-sling" complex in unilateral pulmonary agenesis. Case report. Clin Imaging 1991; 15: $59-61$

86 Kumar B, Kandpal DK, Sharma C et al. Right lung agenesis. Afr J Paediatr Surg 2008; 5: $102-104$

87 Schechter DC. Congenital absence or deficiency of lung tissue. The congenital subtractive bronchopneumonic malformations. Ann Thorac Surg 1968; 6: 287-313

88 Booth JB, Berry CL. Unilateral pulmonary agenesis. Arch Dis Child 1967; 42: $361-374$

89 Döhlemann C, Mantel K, Schneider K et al. Deviated trachea in hypoplasia and aplasia of the right lung: airway obstruction and its release by aortopexy. J Pediatr Surg 1990; 25: 290 - 293

90 Krivchenya DU, Rudenko EO, Lysak SV et al. Lung aplasia: anatomy, history, diagnosis and surgical management. Eur J Pediatr Surg 2007; 17: $244-250$

91 Kravitz RM. Congenital malformations of the lung. Pediatr Clin North Am 1994; 41: 453-472

92 Dressler F, Brandt H-J, Schäfer H. Lungenagenesie und Lungenhypoplasie. Z Kinder-Heilk 1966; 97: 185-194

93 Boxer RA, Hayes $C J$, Hordof $A J$ et al. Agenesis of the left lung and total anomalous pulmonary venous connection. Hemodynamic studies before and after complete surgical correction. Chest 1978; 74: 106 - 109 